Matsushima, Y.

Osaka J. Math.

8 (1971), 1-13

\title{
VECTOR BUNDLE VALUED HARMONIC FORMS AND IMMERSIONS OF RIEMANNIAN MANIFOLDS
}

\author{
Yozo MATSUSHIMA
}

(Received June 1, 1970)

The purpose of this paper is to discuss an application of the theory of vector bundle valued harmonic forms on a Riemannian manifold to the study of immersions.

Let $M$ be a Riemannian manifold and $E$ a Riemannian vector bundle over $M$. Then we can define in a natural way the Laplacian $\square$ operating on $E$-valued differential forms and we can express the scalar product $\langle\square \theta, \theta\rangle$, where $\theta$ is an $E$-valued $p$-form, in terms of curvature and covariant differentials. Moreover, if $M$ is compact, we obtain, by integrating over $M$, a formula analogous to Bochner's for ordinary (i.e. real valued) differential forms.

Let $f$ be an immersion of $M$ into a Riemannian manifold $M^{\prime}$. We may regard the second fundamental form $\alpha$ of $(M, f)$ as a Hom $(T(M), N(M))$-valued 1-form. Assuming that $M^{\prime}$ is of constant sectional curvature, we shall prove that the second fundamental form $\alpha$ is harmonic, i.e. $\square \alpha=0$, if the mean curvature normal of $(M, f)$ is parallel. In particular, if the immersion $f$ is a minimal immersion, then $\alpha$ is harmonic. Conversely, if $M$ is compact and if $\alpha$ is harmonic, then the mean curvature normal is parallel. We obtain from this result together with the formula of Bochner type the results of Simons [5], Chern [1], Nomizu-Smyth [4] and Erbacher [2] proved by them in different ways. In a future paper we shall discuss the case where $M$ is a Kähler manifold.

1. Let $M$ be an $n$-dimensional Riemannian manifold and $E$ a vector bundle over $M$ with a metric along the fibers and a covariant differentiation $D_{X}$ satisfying

$$
X\langle\varphi, \psi\rangle=\left\langle D_{X}, \varphi\right\rangle+\left\langle\varphi, D_{X} \psi\right\rangle
$$

for any vector field $X$ and any sections $\varphi$ and $\psi$ of $E$. A vector bundle $E$ with these properties will be called a Riemannian vector bundle.

We shall denote $C^{p}(E)$ the real vector space of all $E$-valued differential $p$ forms on $M$. We define an operator

$$
\partial: C^{p}(E) \rightarrow C^{p+1}(E),(p=0,1, \cdots)
$$


by the formula

$$
\begin{aligned}
(\partial \theta)\left(X_{1}, \cdots, X_{p+1}\right)= & \sum_{i=1}^{p+1}(-1)^{i+1} D_{X_{i}}\left(\theta\left(X_{1}, \cdots, \hat{X}_{i}, \cdots, X_{p+1}\right)\right) \\
& +\sum_{i<j}(-1)^{i+j} \theta\left(\left[X_{i}, X_{j}\right], X_{1}, \cdots, \hat{X}_{i}, \cdots, \hat{X}_{j}, \cdots, X_{p+1}\right),
\end{aligned}
$$

where $X_{i}$ 's denote vector fields on $M$. The covariant derivative $D_{X} \theta$ of $\theta \in C^{p}(E)$ is an $E$-valued $p$-form such that

$$
\left(D_{X} \theta\right)\left(X_{1}, \cdots, X_{p}\right)=D_{X}\left(\theta\left(X_{1}, \cdots, X_{p}\right)\right)-\sum_{i=1}^{p} \theta\left(X_{1}, \cdots, \nabla_{X} X_{i}, \cdots, X_{p}\right),
$$

where $\nabla_{X} X_{i}$ denotes the covariant derivative of the vector field $X_{i}$ in the Riemannian manifold $M$.

For an $E$-valued 1 -form $\theta$ we have the formula

$$
(\partial \theta)(X, Y)=\left(D_{X} \theta\right)(Y)-\left(D_{Y} \theta\right)(X)
$$

The covariant differential $D \theta$ of $\theta$ is an $E$-valued $(p+1)$-tensor defined by

$$
(D \theta)\left(X_{1}, \cdots, X_{p}, X\right)=\left(D_{X} \theta\right)\left(X_{1}, \cdots, X_{p}\right) .
$$

We define an operator

$$
\partial^{*}: C^{p}(E) \rightarrow C^{p-1}(E) \quad(p>0)
$$

as follows. Let $x \in M$ and let $\left\{e_{1}, \cdots, e_{n}\right\}$ be an orthonormal basis of the tangent space $T_{x}(M)$ of $M$ at $x$. For any $p-1$ tangent vectors $u_{1}, \cdots, u_{p-1}$ at $x$, put

$$
\left(\partial^{*} \eta\right)_{x}\left(u_{1}, \cdots, u_{p-1}\right)=-\sum_{k=1}^{n}\left(D_{e_{k}} \eta\right)_{x}\left(e_{k}, u_{1}, \cdots, u_{p-1}\right)
$$

where $\left(D_{e_{k}} \eta\right)_{x}$ denotes the value of $D_{X} \eta$ at $x$ for any vector field $X$ such that $X_{x}=$ $e_{k}$. Then $\left(\partial^{*} \eta\right)_{x}$ is an alternating $(p-1)$-linear map of $T_{x}(M)$ into $E_{x}$, the fiber of $E$ over $x$, and the assignment $x \rightarrow\left(\partial^{*} \eta\right)_{x}$ defines an $E$-valued $(p-1)$-form $\partial^{*} \theta$. For any $E$-valued 0 -form $\theta$, we define $\partial^{*} \theta=0$.

The Laplacian $\square$ for $E$-valued differential forms is defined as

$$
\square=\partial \partial^{*}+\partial^{*} \partial .
$$

The curvature $\widetilde{R}$ of the covariant differentiation $D$ in $E$ is a $\operatorname{Hom}(E, E)-$ valued 2 -forms given by

$$
\widetilde{R}(X, Y) \varphi=D_{X}\left(D_{Y} \varphi\right)-D_{Y}\left(D_{X} \varphi\right)-D_{[X, Y]} \varphi
$$

for any section $\varphi$ of $E$ and for any vector fields $X$ and $Y$ in $M$. We shall denote by $\langle\theta, \eta\rangle$ the scalar product of two $E$-valued $p$-forms, that is, $\langle\theta, \eta\rangle$ is the smooth function on $M$ given by 


$$
\langle\theta, \eta\rangle(x)=\sum_{i_{1}, \ldots, i_{p}=1}^{n}\left\langle\theta\left(e_{i_{1}}, \cdots, e_{i_{p}}\right), \eta\left(e_{i_{1}}, \cdots, e_{i_{p}}\right)\right\rangle,
$$

where $\left\{e_{1}, \cdots, e_{n}\right\}$ denotes an orthonormal basis of $T_{x}(M)$.

Now we prove the following

Theorem 1. Let $\theta$ be an E-valued 1-form. Then

$$
\langle\square \theta, \theta\rangle=\frac{1}{2} \Delta\langle\theta, \theta\rangle+\langle D \theta, D \theta\rangle+A,
$$

where $\Delta$ denotes the Laplacian of the Riemannian manifold $M$ and $A$ denotes a smooth function in $M$ defined as follows:

$$
A(x)=\sum_{i, j}\left\langle\left(\widetilde{R}\left(e_{j}, e_{i}\right) \theta\left(e_{j}\right), \theta\left(e_{i}\right)\right\rangle+\sum_{i}\left\langle\theta\left(S\left(e_{i}\right)\right), \theta\left(e_{i}\right)\right\rangle,\right.
$$

where $\left\{e_{1}, \cdots, e_{n}\right\}$ is an orthonormal basis of $T_{x}(M)$ and $S$ denotes the endomorphism of $T_{x}(M)$ defined by the Ricci tensor $S$ of $M$, i.e. $S\left(e_{i}\right)=\sum_{k} S_{k i} e_{k}$.

Proof. Fix a point $x \in M$ and let $\left\{e_{1}, \cdots, e_{n}\right\}$ be an orthonormal basis of $T_{x}(M)$. We can choose $n$ vector fields $E_{1}, \cdots, E_{n}$ in $M$ such that $E_{i}(x)=e_{i}$ and $\left(\nabla_{E_{k}}\right.$ $\left.E_{i}\right)_{x}=0$ for $i, k=1, \cdots, n$. Then, because $\nabla_{e_{s}} E_{i}$ are zero for $i, s=1, \cdots, n$, we have

$$
\begin{aligned}
(\partial * \partial \theta)\left(e_{i}\right) & =-\sum_{s}\left(D_{e_{s}} \partial \theta\right)\left(e_{s}, e_{i}\right)=-\sum_{s} D_{e_{s}}\left((\partial \theta)\left(E_{s}, E_{i}\right)\right) \\
& =-\sum_{s} D_{e_{s}}\left(\left(D_{E_{s}} \theta\right)\left(E_{i}\right)-\left(D_{E_{i}} \theta\right)\left(E_{s}\right)\right) \\
& =\sum_{s}\left(D_{E_{s}} D_{E_{i}} \theta\right)\left(e_{i}\right)-\sum_{s}\left(D_{E_{s}} D_{E_{s}} \theta\right)\left(e_{i}\right) .
\end{aligned}
$$

On the other hand, $\partial^{*} \theta=-\sum_{s, t} g^{s t}\left(D_{E_{t}} \theta\right)\left(E_{s}\right)$ where $\left(g^{s t}\right)$ is the inverse matrix of the matrix $\left(g\left(E_{s}, E_{t}\right)\right)$, we have

$$
\begin{aligned}
\left(\partial \partial^{*} \theta\right)\left(e_{i}\right) & =D_{e_{i}}\left(\partial^{*} \theta\right)=-\sum_{s, t}\left(e_{i} g^{s t}\right)\left(D_{e_{t}} \theta\right)\left(e_{s}\right)-\sum_{s, t} \delta^{s t} e_{i}\left(\left(D_{E_{t}} \theta\right)\left(E_{s}\right)\right) \\
& =-\sum_{s} e_{i}\left(\left(D_{E_{s}} \theta\right)\left(E_{s}\right)\right)=-\sum_{s}\left(D_{E_{i}} D_{E_{s}} \theta\right)\left(e_{s}\right),
\end{aligned}
$$

because $\nabla_{e_{i}} E_{k}=0$ at $x$.

Therefore we obtain

$$
(\square \theta)\left(e_{i}\right)=\sum_{s}\left(\left(D_{E_{s}} D_{E_{i}}-D_{E_{i}} D_{E_{s}}\right) \theta\right)\left(e_{s}\right)-\sum_{s}\left(D_{E_{s}} D_{E_{s}} \theta\right)\left(e_{i}\right) .
$$

Since $\left[E_{s}, E_{i}\right]=0$ at $x$, we have

$$
\begin{aligned}
\left(\left(D_{E_{s}} D_{E_{i}}-D_{E_{i}} D_{E_{s}}\right) \theta\right)\left(e_{s}\right) & =\left(\left(\left[D_{E_{s}}, D_{E_{i}}\right]-D_{\left[E_{s}, E_{i}\right]}\right) \theta\right)\left(e_{s}\right) \\
& =\widetilde{R}\left(e_{s}, e_{i}\right)\left(\theta\left(e_{s}\right)\right)-\theta\left(R\left(e_{s}, e_{i}\right) e_{s}\right)
\end{aligned}
$$

Therefore 


$$
\begin{aligned}
\langle\square \theta, \theta\rangle & =\sum_{i}\left\langle(\square \theta) \theta\left(e_{i}\right),\left(e_{i}\right)\right\rangle \\
& =\sum_{s, t}\left\langle\widetilde{R}\left(e_{s}, e_{i}\right) \theta\left(e_{s}\right), \theta\left(e_{i}\right)\right\rangle+\sum_{i} \theta\left(S\left(e_{i}\right), \theta\left(e_{i}\right)\right) \\
& -\sum_{s, t}\left\langle\left(D_{E_{s}} D_{E_{s}} \theta\right)\left(e_{i}\right), \theta\left(e_{i}\right)\right\rangle .
\end{aligned}
$$

Now by a local computation we see that

$$
\begin{aligned}
& -\sum_{s, i}\left\langle\left(D_{E_{s}} D_{E_{s}} \theta\right)\left(e_{i}\right), \theta\left(e_{i}\right)\right\rangle \\
& =\langle D \theta, D \theta\rangle(x)+\frac{1}{2}(\Delta\langle\theta, \theta\rangle)(x) .
\end{aligned}
$$

Thus we have proved that

$$
\langle\square \theta, \theta\rangle=\frac{1}{2} \Delta\langle\theta, \theta\rangle+\langle D \theta, D \theta\rangle+A .
$$

Corollary 1. Let $\theta$ be an E-valued 1-form. Assume that $\square \theta=0$ and $\Delta\langle\theta, \theta\rangle=0$. Then we have $A \leqq 0$ everywhere on $M$.

Assume now that $M$ is compact and oriented. Then we can define the inner product $(\theta, \eta)$ of two $E$-valued $p$-forms by

$$
(\theta, \eta)=\int_{M}\langle\theta, \eta\rangle * 1
$$

Then we obtain from Theorem 1 the following corollary.

Corollary 2. Let $\theta$ be an $E$-valued 1-form such that $\square \theta=0$. Then we have

$$
(D \theta, D \theta)+\int_{M} A * 1=0 .
$$

If $A \geqq 0$ everywhere on $M$, then we have $A \equiv 0$ and $D \theta=0$.

We remark that the operator $\partial^{*}$ is the adjoint operator of $\partial$, i.e.

$$
(\partial \theta, \eta)=\left(\theta, \partial^{*} \eta\right)
$$

for any $\theta \in C^{p}(E)$ and $\eta \in C^{p+1}(E)$ and hence we have

$$
(\square \theta, \theta)=(\partial \theta, \partial \theta)+\left(\partial^{*} \theta, \partial^{*} \theta\right) .
$$

Therefore, if $M$ is compact, $\square \theta=0$ if and only if $\partial \theta=0$ and $\partial^{*} \theta=0$.

2. Let $M$ be an $n$-dimensional Riemannian manifold isometrically immersed in a Riemannian manifold $M^{\prime}$ of dimension $n+p$. We shall denote by $N(M)$ and $\alpha$ the normal bundle and the second fundamental form of $M$ [3]. The second fundamental form $\alpha$ is an $N(M)$-valued symmetric 2-form on $M$.

In the following we put 


$$
E=\operatorname{Hom}(T(M), N(M))=T^{*}(M) \otimes N(M)
$$

and we interprete $\alpha$ as an $E$-valued 1-form $\beta$ as follows: For any vector field $X$ in $M, \beta(X)$ is a section of $E$ such that

$$
\beta(X) \cdot Y=\alpha(X, Y)
$$

for all vector field $Y$ in $M$. Then we have

$$
\beta(X) \cdot Y=\beta(Y) \cdot X .
$$

We call also $\beta$ the second fundamental form of $M$.

A metric along the fibres of $E$ is defined naturally by the Riemann metrics of $M$ and $M^{\prime}$ and a covariant derivation $D_{X}$ in $E$ is also naturally defined by the covariant differentiation $\nabla_{X}$ in $M$ and $D_{\bar{X}}^{\perp}$ in $N(M)$, where for any normal vector $\xi$ of $M, D_{X}^{\perp} \xi$ is defined as the normal component of $\nabla_{X}{ }^{\prime} \xi$, where $\nabla_{X}{ }^{\prime}$ denote the covariant differentiation in the Riemannian manifold $M^{\prime}$ (See [3]).

Let $\varphi$ be a section of $E$. We may regard $\varphi$ as an $N(M)$-valued 1-form on $M$ and we have

$$
\begin{aligned}
& \left(D_{X} \varphi\right)(Y)=D_{\bar{X}}^{\perp}(\varphi(Y))-\varphi\left(\nabla_{X} Y\right), \\
& \left\langle D_{X} \varphi, \psi\right\rangle+\left\langle\varphi, D_{X} \psi\right\rangle=X\langle\varphi, \psi\rangle
\end{aligned}
$$

for any sections $\varphi$ and $\psi$ of $E$.

The following Proposition 1 may be considered as an interpretation of the equation of Codazzi in our formalism.

Proposition 1. Assume that $M^{\prime}$ is a Riemannian manifold of constant sectional curvature. Then the second fundamental form $\beta$ of $M$ satisfies the equation $\partial \beta=0$.

Proof. By a straightforward computation we see that

$$
\begin{aligned}
(\partial \beta(X, Y))(Z) & =\left\{D_{\bar{X}}^{\perp}(\alpha(Y, Z))-\alpha\left(\nabla_{X} Y, Z\right)-\alpha\left(Y, \nabla_{X} Z\right)\right\} \\
& -\left\{D_{\bar{Y}}^{\perp}(\alpha(X, Z))-\alpha\left({ }_{Y} X, Z\right)-\alpha\left(X, \nabla_{Y} Z\right)\right\}
\end{aligned}
$$

and the right hand side is 0 by [3, Vol. II, P. 25, Cor. 4.4].

For each normal vector $\nu \in N_{X}(M)$ we define an endomorphism $A_{\nu}$ of $T_{x}(M)$ by the formula

$$
\left\langle A_{\nu}(u), v\right\rangle=\langle\beta(u) v, \nu\rangle
$$

for any tangent vectors $u, v \in T_{x}(M)$. The mean curvature normal $\eta$ of $M$ is a 
normal vector field in $M$ such that

$$
\frac{1}{n} \operatorname{Tr} A_{\nu}=\langle\nu, \eta(x)\rangle
$$

for any $\nu \in N_{x}(M)$ and $x \in M$.

$M$ is said to be minimal in $M^{\prime}$ if the mean curvature normal vanishes at each point, that is, if $\operatorname{Tr} A_{\nu}=0$ for any $\nu \in N_{x}(M)$ and $x \in M$.

We say that $M$ has a constant mean curvature if the mean curvature normal $\eta$ is parallel, that is, $D_{X}^{\perp} \eta=0$ for any vector field $X$ in $M$.

Let $\nu$ be a normal vector field. Then we have $\operatorname{Tr} \mathrm{A}_{\nu}=n\langle\nu, \eta\rangle$ and hence $X \cdot \operatorname{Tr} A_{\nu}=n\left\{\left\langle D_{X}^{\frac{1}{X}} \nu, \eta\right\rangle+\left\langle\nu, D_{X}^{\frac{1}{X}} \eta\right\rangle\right\}$. Therefore $M$ has a constant mean curvature, if and only if

$$
X \cdot \operatorname{Tr} A_{\nu}=\operatorname{Tr} A_{D_{X}^{\perp}}
$$

for any normal vector field $\nu$ and any vector field $X$ in $M$.

Proposition 2. Let $M^{\prime}$ be a Riemmanian manifold of constant sectional curvature. Then the second fundamental form $\beta$ of $M$ satisfies the equation $\partial^{*} \beta=0$ if and only if $M$ has a constant mean curvature.

Proof. Let $x$ be a point in $M$ and let $\left\{e_{1}, \cdots, e_{n}\right\}$ be an orthonormal basis of $T_{x}(M)$. Let $E_{1}, \cdots, E_{n}$ be vector fields in a neighborhood of $x$ such that $\left(E_{i}\right)_{x}=e_{i}$ and $\nabla_{E_{i}} E_{k}=0$ at $x$ for $i, k=1, \cdots, n$. $\operatorname{Let}\left(g^{s t}\right)$ the inverse matrix of the matrix $\left(\left\langle E_{s}, E_{t}\right\rangle\right)$. Then $\partial^{*} \beta=-g^{s t}\left(D_{E_{t}} \beta\right)\left(E_{s}\right)^{1)}$ and $\left(\partial^{*} \beta\right) \cdot E_{k}=-g^{s t}\left(D_{E_{t}} \beta\right)\left(E_{s}\right) \cdot E_{k}$. $\operatorname{Since}\left(D_{E_{t}} \beta\right)\left(E_{s}\right) E_{k}=D_{E_{t}}^{\perp}\left(\alpha\left(E_{s}, E_{k}\right)\right)-\alpha\left(\nabla_{E_{t}} E_{s}, E_{k}\right)-\alpha\left(E_{s}, \nabla_{E_{t}} E_{k}\right)$ and since $\alpha$ is symmetric, we get $\left(D_{E_{t}} \beta\right)\left(E_{s}\right) E_{k}=\left(D_{E_{t}} \beta\right)\left(E_{k}\right) E_{s}$. On the other hand, by Proposition 1, we have $\partial \beta=0$ and hence $\left(D_{E t} \beta\right)\left(E_{k}\right)=\left(D_{E_{k}} \beta\right)\left(E_{t}\right)$, hence $\left(D_{E_{t}} \beta\right)\left(E_{s}\right) E_{k}=\left(D_{E_{k}} \beta\right)\left(E_{t}\right) \cdot E_{s}$. Therefore, for any normal vector field $\nu$, we have

$$
\begin{aligned}
&\left\langle\left(\partial^{*} \beta\right) \cdot E_{k}, \nu\right\rangle=-g^{s t}\left\langle\left(D_{E_{k}} \beta\right)\left(E_{t}\right) E_{s}, \nu\right\rangle \\
&=-g^{s t}\left\{\left\langle D_{E_{k}}^{\perp}\left(\alpha\left(E_{t}, E_{s}\right)\right), \nu\right\rangle\right.-\left\langle\alpha\left(\nabla_{E_{k}} E_{t}, E_{s}\right), \nu\right\rangle \\
&\left.-\left\langle\alpha\left(E_{t}, \nabla_{E_{k}} E_{s}\right), \nu\right\rangle\right\} .
\end{aligned}
$$

Now

$$
\begin{aligned}
& g^{s t}\left\langle D_{E_{k}}^{\perp}\left(\alpha\left(E_{t}, E_{s}\right), \nu\right\rangle\right. \\
= & g^{s t}\left\{E_{k}\left\langle\alpha\left(E_{t}, E_{s}\right), \nu\right\rangle-\left\langle\alpha\left(E_{t}, E_{s}\right), D_{E_{k}}^{\perp} \nu\right\rangle\right. \\
= & E_{k}\left(g^{s t}\left\langle\alpha\left(E_{t}, E_{s}\right), \nu\right\rangle\right)-\left(E_{k} g^{s t}\right)\left\langle\alpha\left(E_{t}, E_{s}\right), \nu\right\rangle-g^{s t}\left\langle\alpha\left(E_{t}, E_{s}\right), D_{E_{k}}^{\perp} \nu\right\rangle \\
= & E_{k}\left(T_{r} A_{\nu}\right)-T_{r} A_{D_{E_{k}}^{\perp}}-E_{k} g^{s t} \cdot\left\langle\alpha\left(E_{t}, E_{s}\right), \nu\right\rangle .
\end{aligned}
$$

1) We omit here the summation signs. 
Since $\nabla_{E_{k}} E_{i}=0$ at $x$, we have $E_{k} g^{s t}=0$ at $x$. Therefore we get from the above that

$$
\left\langle\left(\partial^{*} \beta\right) E_{k}, \nu\right\rangle(x)=\operatorname{Tr} A_{D_{E}^{\nu}}-E_{k}\left(\operatorname{Tr} A_{\nu}\right)
$$

at $x$ for $k=1,2, \cdots, n$ and hence for any vector field $X$ we have $\left\langle\left(\partial^{*} \beta\right) X, \nu\right\rangle(x)$ $=\operatorname{Tr} A_{D^{\frac{1}{X}}}-X\left(\operatorname{Tr} A_{\nu}\right)$ at $x$. Since $x$ is an arbitrary point of $M$ and $\nu$ is an arbitrary normal vector field, we see from the above equation that $\partial^{*} \beta=0$ if and only if $M$ has a constant mean curvature.

From Propositions 1 and 2 we get the following

Theorem 2. Let $M$ be a Riemannian manifold immersed isometrically into a Riemannian manifold $M^{\prime}$ of constant sectional curvature. Let $\beta$ be the second fundamental form of $M$ regarded as a Hom $(T(M), N(M))$-valued 1-form. Then $\beta$ satisfies the equation $\square \beta=0$, if $M$ has a constant mean curvature. Conversely, if $M$ is compact and orientable and $\square \beta=0$, then $M$ has a constant mean curvature.

3. We shall discuss in this section some applications of Theorems 1 and 2. Let $M$ be a Riemannian manifold immersed isometrically into a Riemannian manifold $M^{\prime}$ of constant sectional curvature $c$. Let $x \in M$ and let $\left\{e_{1}, \cdots, e_{n}\right\}$ and $\left\{\nu_{1}, \cdots, \nu_{p}\right\}$ be orthonormal bases of $T_{x}(M)$ and $N_{x}(M)$ respectively. We shall denote by $A_{a}(a=1,2, \cdots, p)$ the endomorphism of $T_{x}(M)$ defined by $\left\langle A_{a} u, v\right\rangle=$ $\left\langle\beta(u) \cdot v, \nu_{a}\right\rangle$ and put $A_{a} \cdot e_{i}=\sum_{j}\left(A_{a}\right)_{i}^{j} e_{j}$. Then we have the following Gauss equation:

$$
R_{k l i j}=c\left\{\delta_{k i} \delta_{l j}-\delta_{k j} \delta_{l i}\right\}+\sum_{a}\left\{\left(A_{a}\right)_{i}^{k}\left(A_{a}\right)_{i}^{l}-\left(A_{a}\right)_{j}^{k}\left(A_{a}\right)_{i}^{l}\right\}
$$

where $R_{k l i j}$ denote the components of the curvature tensor with respect to the basis $\left\{e_{1}, \cdots, e_{n}\right\}$ of $T_{x}(M)$. Then the endomorphism $S$ of $T_{x}(M)$ defined by $S\left(e_{j}\right)$ $=\sum_{l} S_{l j}\left(e_{l}\right)$ with $S_{e j}=\sum_{k} R_{k l k j}$ is of the form

$$
S=c(n-1) I+\sum_{a}\left(\operatorname{Tr} A_{a}\right) A_{a}-\sum_{a} A_{a}^{2},
$$

where $I$ denotes the identity endomorphism of $T_{x}(M)$.

Let $K$ be the scalar curvature of $M$. Then $K(x)=\operatorname{Tr} \mathrm{S}=c(n-1) n+$ $\sum_{a}\left(\operatorname{Tr} A_{a}\right)^{2}-\sum_{a} \operatorname{Tr} A_{a}^{2}$. The value $\eta(x)$ at $x$ of the mean curvature normal $\eta$ is given by $\eta(x)=\frac{1}{n} \sum_{a} \operatorname{Tr} A_{a} \cdot \nu_{a}$ and hence $n^{2}\langle\eta, \eta\rangle(x)=\sum\left(\operatorname{Tr} A_{a}\right)^{2}$. Analogously we have $\langle\beta, \beta\rangle(x)=\sum_{a} \operatorname{Tr} A_{a}^{2}$. Hence we get

$$
K=c(n-1) n+n^{2}\langle\eta, \eta\rangle-\langle\beta, \beta\rangle,
$$


where $\beta$ and $\eta$ denotes the second fundamental form and the mean curvature normal of $M$ respectively. For any Riemannian vector bundle $E$ over $M$ we have defined the endomorphism $\widetilde{R}(u, v)$ of the fiber $E_{x}$, where $u, v \in T_{x}(M)$. Let $E=$ $\operatorname{Hom}\left(T(M), N(M)\right.$ and let $\varphi \in E_{x}$. Then $\widetilde{R}(u, v) \varphi$ is an element of $E_{x}=$ Hom $\left(T_{x}(M), N_{x}(M)\right)$ such that

$$
\left.(\widetilde{R}(u, v) \varphi)(w)=R^{\perp}(u, v)(w) \varphi\right)-\varphi(R(u, v) w),
$$

where $u, v, w \in T_{x}(M)$ and $R^{\perp}$ denotes the curvature of the Riemannian vector bundle $N(M)$.

Let $\nu$ be a normal vector of $M$ at $x$ and let $N$ be a normal vector field such that $N_{x}=\nu$. Let $X$ and $Y$ be vector fields in $M$ such that $X_{x}=u$ and $Y_{x}=v$. Then we have

$$
R^{\perp}(u, v) \nu=\left(D_{\bar{X}}^{\perp} D_{Y}^{\perp}-D_{Y}^{\perp} D_{X}^{\perp}-D^{\perp}{ }_{[X, Y]}\right) N
$$

at $x$.

Denote by $\nabla^{\prime}$ the covariant derivation in the ambiant space $M^{\prime}$. Then we have

$$
\begin{aligned}
& \nabla_{X}^{\prime} Y=\nabla_{X} Y+\alpha(X, Y), \\
& \nabla_{X}^{\prime} N=-A_{N}(X)+D_{X}^{\perp} N .
\end{aligned}
$$

We see from these two equations that the normal component $\left(R^{\prime}(X, Y) N\right)^{\perp}$ of $R^{\prime}$ $(X, Y) N$, where $R^{\prime}$ denotes the curvature tensor of $M_{i}$, is equal to $R^{\perp}(X, Y) N$ $-\alpha\left(A_{N}(Y), X\right)+\alpha\left(A_{N}(X), Y\right)$. Since $M^{\prime}$ is of constant curvarute $R^{\prime}(X, Y) N$ $=c\{\langle N, Y\rangle X-\langle N, X\rangle Y\}=0$ and hence we get $R^{\perp}(X, Y) N=-\alpha\left(A_{N}(X), Y\right)$ $+\alpha\left(A_{N}(Y), X\right)$. Thus we have

$$
R^{\perp}(u, v) \nu=-\alpha\left(A_{\imath} u, v\right)+\alpha\left(A_{\nu} v, u\right) .
$$

In particular

$$
R^{\perp}(u, v) \nu_{a}=-\alpha\left(A_{a} u, v\right)+\alpha\left(u, A_{a} v\right) .
$$

Since $\alpha\left(A_{a} u, v\right)=\sum_{b}\left\langle\alpha\left(A_{a} u, v\right), \nu_{b}\right\rangle \nu_{b}=\sum_{b}\left(A_{b} A_{a} u, v\right) \nu_{b}$ and $\alpha\left(u, A_{a} v\right)=\sum_{b}\left\langle A_{b} u, A_{a} v\right\rangle \nu_{b}=\sum_{b}\left\langle A_{a} A_{b} u, v\right\rangle \nu_{b}$ we get

$$
R^{\perp}(u, v) \nu_{a}=\sum_{b}\left\langle\left[A_{a}, A_{b}\right] u, v\right\rangle \nu_{b}
$$

Now by Theorem 1 , we have

$$
\langle\square \beta, \beta\rangle=\frac{1}{2}+\Delta\langle\beta, \beta\rangle+\langle D \beta, D \beta\rangle+A,
$$


where

$$
A(x)=\sum_{i, j}\left\langle\widetilde{R}\left(e_{j}, e_{i}\right) \beta\left(e_{j}\right), \beta\left(e_{i}\right)\right\rangle+\sum_{i}\left\langle\beta\left(S\left(e_{i}\right), \beta\left(e_{i}\right)\right\rangle .\right.
$$

Now

$$
\begin{aligned}
& \sum_{i}\left\langle\beta\left(S\left(e_{i}\right), \beta\left(e_{i}\right)\right\rangle=\sum_{i, j}\left\langle\alpha\left(S\left(e_{i}\right), e_{j}\right), \alpha\left(e_{i}, e_{j}\right)\right\rangle\right. \\
& =\sum_{i, j, a}\left\langle A_{a}\left(S\left(e_{i}\right)\right), e_{j}\right\rangle\left\langle A_{a}\left(e_{i}\right), e_{j}\right\rangle=\sum_{a} \operatorname{Tr}\left(S A_{a}^{2}\right)
\end{aligned}
$$

and by (3.2) we get

$$
\begin{gathered}
\sum_{i}\left\langle\beta\left(S\left(e_{i}\right), \beta\left(e_{i}\right)\right\rangle\right. \\
=c(n-1) \sum_{a} \operatorname{Tr} A_{a}^{2}+\sum_{a, b} \operatorname{Tr} A_{a} \cdot \operatorname{Tr}\left(A_{a} A_{b}^{2}\right)-\sum_{a, b} \operatorname{Tr}\left(A_{a}^{2} A_{b}^{2}\right) .
\end{gathered}
$$

On the other hand,

$$
\begin{aligned}
& \sum_{i, j}\left\langle\widetilde{R}\left(e_{j}, e_{i}\right) \beta\left(e_{j}\right), \beta\left(e_{i}\right)\right\rangle \\
= & \sum_{i, j, k}\left\langle R^{\perp}\left(e_{j}, e_{i}\right) \alpha\left(e_{j}, e_{k}\right), \alpha\left(e_{i}, e_{k}\right)\right\rangle-\sum_{i, j, k}\left\langle\alpha\left(e_{j}, R\left(e_{j}, e_{i}\right) e_{k}\right), \alpha\left(e_{i}, e_{k}\right)\right\rangle \\
= & \sum_{i, j, k} \sum_{a, b}\left\langle A_{a} e_{j}, e_{k}\right\rangle\left\langle A_{b} e_{i}, e_{k}\right\rangle\left\langle R^{\perp}\left(e_{j}, e_{i}\right) \nu_{a} \nu_{b}\right\rangle \\
- & \sum_{i, j, k} \sum_{a}\left\langle A_{a} e_{j}, R\left(e_{j}, e_{i}\right) e_{k}\right\rangle\left\langle A_{a} e_{i}, e_{k}\right\rangle .
\end{aligned}
$$

and by (3.5), the first term equals $\sum_{a, b} \operatorname{Tr}\left(A_{a} A_{b}\left[A_{a}, A_{b}\right]\right)=-\sum_{a, b} \operatorname{Tr}\left(A_{a}^{2} A_{a}^{2}\right)+\sum_{a, b}$ $\operatorname{Tr}\left(A_{a} A_{b}\right)^{2}$ and by the Gauss equation (3.1) the second term equals $-c \sum_{a}(\operatorname{Tr}$ $\left.A_{a}\right)^{2}+c \sum_{a} \operatorname{Tr}\left(A_{a}^{2}\right)-\sum_{a, b}\left(\operatorname{Tr}\left(A_{a} A_{b}\right)\right)^{2}+\sum_{a, b} \operatorname{Tr}\left(A_{a} A_{b}\right)^{2}$.

Therefore we have

$$
\begin{aligned}
& \sum_{i, j}\left\langle\widetilde{R}\left(e_{u}, e_{i}\right) \beta\left(e_{j}\right), \beta\left(e_{i}\right)\right\rangle \\
= & c \sum_{a} \operatorname{Tr} A_{a}^{2}-c \sum_{v}\left(\operatorname{Tr} A_{a}\right)^{2}-\sum_{a, b} \operatorname{Tr}\left(A_{a}^{2} A_{a}^{2}\right)-\sum_{a, b}\left(\operatorname{Tr}\left(A_{a} A_{b}\right)\right)^{2}+ \\
& +2 \sum_{a, b} \operatorname{Tr}\left(A_{a} A_{b}\right)^{2}
\end{aligned}
$$

Then we get from (3.6), (3.7) and (3.8) that

$$
\begin{gathered}
A(x)=c n \sum_{a} \operatorname{Tr} A_{b}^{2}-c \sum_{a}\left(\operatorname{Tr} A_{a}\right)^{2}-\sum_{a, b}\left(\operatorname{Tr}\left(A_{a} A_{b}\right)\right)^{2} \\
+\sum_{a, b} \operatorname{Tr} A_{a} \cdot \operatorname{Tr}\left(A_{a} A_{b}^{2}\right)+\sum_{a, b} \operatorname{Tr}\left[A_{a}, A_{b}\right]^{2} .
\end{gathered}
$$

Now let $\lambda_{1}^{(a)}, \cdots, \lambda_{n}^{(a)}$ be eigen-values of $A_{a}$ and let $\left\{e_{1}^{(a)}, \cdots, e_{n}^{(n)}\{\right.$ be an orthonormal basis of $T_{x}(M)$ such that $A_{a} e_{i}^{(a)}=\lambda_{i}^{(a)} e_{i}^{(a)}(i=1, \cdots, n, a=1, \cdots, p)$.

We shall denote by $K_{i j}^{(a)}$ the sectional curvature for the 2-plane spanned by $e_{i}^{(a)}$ and $e_{j}^{(a)}, i \neq j$.

We show that 


$$
A(x)=\sum_{v} \sum_{i<j}\left(\lambda_{i}^{(a)}-\lambda_{j}^{(a)}\right)^{2} K_{i j}^{(a)}+\frac{1}{2} \sum_{a, b} \operatorname{Tr}\left[A_{a}, A_{b}\right]^{2} .
$$

We write $A(x)$ in the following form:

$$
\begin{aligned}
& A(x)=B(x)+\sum_{a \neq b} \operatorname{Tr} A_{a} \cdot \operatorname{Tr}\left(A_{a} A_{b}^{2}\right)-\sum_{a \neq b}\left(\operatorname{Tr}\left(A_{a} A_{b}\right)\right)^{2}+ \\
& +\sum_{a, b} \operatorname{Tr}\left[A_{a}, A_{b}\right]^{2},
\end{aligned}
$$

where

$$
B(x)=\sum_{a}\left\{c n \operatorname{Tr} A_{a}^{2}-c\left(\operatorname{Tr} A_{a}\right)^{2}-\left(\operatorname{Tr} A_{a}^{2}\right)^{2}+\operatorname{Tr} A_{a} \cdot \operatorname{Tr} A_{a}^{3}\right\} .
$$

Now by a lemma of Nomizu-Smyth [4] we have

$$
\begin{aligned}
& \text { cn } \operatorname{Tr} A_{a}^{2}-c\left(\operatorname{Tr} A_{a}\right)^{2}-\left(\operatorname{Tr} A_{a}^{2}\right)^{2}+\operatorname{Tr} A_{a} \cdot \operatorname{Tr}\left(A_{a}\right)^{3} \\
& =\sum_{i<j}\left(\lambda_{i}^{(a)}-\lambda_{j}^{(a)}\right)^{2}\left(c+\lambda_{j}^{(a)} \lambda_{j}^{(a)}\right)
\end{aligned}
$$

for each $a$. Now fix an index $a$ and let

$$
A_{b} e_{i}^{(a)}=\sum_{j}\left(A_{b}\right)_{i}^{s} e_{j}^{(a)} \quad(b=1,2, \cdots, p)
$$

Then we have $\left(A_{a}\right)_{j}^{t}=\delta_{j}^{i} \lambda_{j}^{(a)}$ and hence

$$
\left(A_{a} A_{b}\right)_{j}^{i}=\lambda_{i}^{(a)}\left(A_{b}\right)_{j}^{i},\left(A_{b} A_{a}\right)_{j}^{i}=\left(A_{b}\right)_{j}^{j} \lambda_{j}^{(a)} .
$$

By the equation of Gauss we have

$$
\begin{aligned}
K_{i j}^{(a)} & =R\left(e_{i}^{(a)}, \mathrm{e}_{j}^{(a)}, e_{i}^{(a)}, e_{j}^{(a)}\right) \\
& =c+\sum_{b}\left(A_{b}\right)_{i}^{i}\left(A_{b}\right)_{j}^{j}-\sum_{b}\left(A_{b}\right)_{j}^{j}\left(A_{b}\right)_{i}^{j} . \\
& =c+\lambda_{i}^{(a)} \lambda_{j}^{(a)}+\sum_{b \neq a}\left(A_{b}\right)_{i}^{i}\left(A_{b}\right)_{j}^{j}-\sum_{b}\left(A_{b}\right)_{j}^{i}\left(A_{b}\right)_{i}^{j} .
\end{aligned}
$$

Hence we have

$$
\begin{aligned}
& \left(\lambda_{i}^{(a)} \lambda-{ }_{j}^{(a)}\right)^{2}\left(c+\lambda_{i}^{(a)} \lambda_{j}^{(a)}\right) \\
= & \left(\lambda_{i}^{(a)}-\lambda_{j}^{(a)}\right)^{2} K_{i j}^{(a)}+\sum_{b}\left(\lambda_{i}^{(a)}-\lambda_{j}^{(a)}\right)^{2}\left(A_{b}\right)_{j}^{i}\left(A_{b}\right)_{i}^{j} \\
- & \sum_{b \neq a}\left(\lambda_{i}^{(a)}-\lambda_{j}^{(a)}\right)^{2}\left(A_{b}\right)_{i}^{i}\left(A_{b}\right)_{j}^{j} .
\end{aligned}
$$

This equality holds also for $i=j$ trivially if we define $K_{i i}^{(a)}=0$.

Then by (3.14)

$$
\begin{aligned}
& \sum_{i<j}\left(\lambda_{i}^{(a)}-\lambda_{j}^{(a)}\right)^{2}\left(c+\lambda_{i}^{(a)} \lambda_{j}^{(a)}\right)=\frac{1}{2} \sum_{i, j}\left(\lambda_{i}^{(a)}-\lambda_{j}^{(a)}\right)^{2}\left(c+\lambda_{i}^{(a)} \lambda_{j}^{(a)}\right) \\
= & \sum_{i<j}\left(\lambda_{i}^{(a)}-\lambda_{j}^{(a)}\right)^{2} K_{i j}^{(a)}-\frac{1}{2} \sum_{b} \sum_{i, j}\left(\lambda_{i}^{(a)}-\lambda_{j}^{(a)}\right)\left(A_{b}\right)_{j}^{i}\left(\lambda_{j}^{(a)}-\lambda_{i}^{(a)}\right)\left(A_{b}\right)_{i}^{j}
\end{aligned}
$$




$$
\begin{aligned}
& \begin{array}{l}
-\frac{1}{2} \sum_{b \neq a}\left\{\sum_{i, j}\left(\lambda_{i}^{(a)}\right)^{2}\left(A_{a}\right)_{i}^{i} \sum_{j}\left(A_{b}\right)_{j}^{j}-2 \sum_{i} \lambda_{i}^{(a)}\left(A_{b}\right)_{i}^{i} \sum_{j} \lambda_{j}^{(a)}\left(A_{b}\right)_{j}^{j}\right. \\
\left.+\sum_{i}\left(A_{b}\right)_{i}^{i} \sum_{j} \lambda_{j}^{(a)}\left(A_{b}\right)_{j}^{j}\right\}
\end{array} \\
& =\sum_{i<j}\left(\lambda_{i}^{(a)}-\lambda_{j}^{(a)}\right)^{2} K_{i j}^{(a)}-\frac{1}{2} \sum_{b} \operatorname{Tr}\left[A_{a}, A_{b}\right]^{2} \\
& -\sum_{b \neq a}\left\{\operatorname{Tr} A_{b} \cdot \operatorname{Tr}\left(A_{a}^{2} A_{b}\right)-\left(\operatorname{Tr}\left(A_{a} A_{b}\right)\right)^{2}\right\} .
\end{aligned}
$$

Then we obtain from (3.11), (3.12) and (3.13) the equality (3.10).

Now we cite the following two lemmas from [1].

Lemma 1. Let $A$ and $B$ be symmetric $n \times n$ matrices. Then

$$
\operatorname{Tr}[A, B]^{2} \geqq-2 \operatorname{Tr} A^{2} \cdot \operatorname{Tr} B^{2},
$$

and the equality holds for non-zero matrices $A$ and $B$ if and only if $A$ and $B$ can be transformed simultaneously by an orthogonal matrix into scalar multiple of $\widehat{A}$ and $\widetilde{B}$ respectively, where

$$
\widehat{A}=\left(\begin{array}{ll|l}
0 & 1 & 0 \\
1 & 0 & 0 \\
\hline 0 & 0
\end{array}\right), \quad \widetilde{B}=\left(\begin{array}{rr|r}
1 & 0 & 0 \\
0 & -1 & \\
\hline 0 & 0
\end{array}\right)
$$

Lemma 2. Let $A_{1}, A_{2}$ and $A_{3}$ be $n \times n$ symmetric matrices and if

$$
\operatorname{Tr}\left[A_{a}, A_{b}\right]^{2}=-2 \operatorname{Tr} A_{a}^{2} \cdot \operatorname{Tr} A_{b}^{2}
$$

for $1 \leqq a<b \leqq 3$, then at least one of the matrices $A_{a}$ must be zero.

By Lemma 1, we have

$$
\frac{1}{2} \sum_{a, b} \operatorname{Tr}\left[A_{a}, A_{b}\right]^{2} \geqq-\sum_{a \neq b} \operatorname{Tr} A_{a}^{2} \cdot \operatorname{Tr} A_{b}^{2}=-2 \sum_{a<b} \operatorname{Tr} A_{a}^{2} \cdot \operatorname{Tr} A_{b}^{2} .
$$

Put $S_{a}=\operatorname{Tr} A_{a}^{2}$. Then $\sum_{a} S_{a}=\langle\beta, \beta\rangle(x)$.

Since

$$
\begin{aligned}
0 \leqq \sum_{a<b}\left(S_{a}-S_{b}\right)^{2} & =\sum_{a<b}\left(S_{a}^{2}+S_{b}^{2}\right)-2 \sum_{a<b} S_{a} S_{b} \\
& =(p-1) \sum_{a} S_{a}^{2}-2 \sum_{a<b} S_{a} S_{b} \\
& =(p-1)\left\{\left(\sum_{a} S_{a}\right)^{2}-2 \sum_{a<b} S_{a} S_{b}\right\}-2 \sum_{a<b} S_{a} S_{b} \\
& =(p-1)\langle\beta, \beta\rangle^{2}(x)-2 p \sum_{a<b} S_{a} S_{b}
\end{aligned}
$$

we have 


$$
-2 \sum_{a<b} S_{a} S_{b} \geqq-\frac{(p-1)}{p}\langle\beta, \beta\rangle^{2}(x)
$$

ane here the equality holds if and only if $S_{a}=S_{b}$ for $a, b=1, \cdots, p$. Therefore we get

$$
\frac{1}{2} \sum_{a, b} \operatorname{Tr}\left[A_{a}, A_{b}\right]^{2} \geqq-\frac{(p-1)}{p}\langle\beta, \beta\rangle^{2}(x)
$$

and the equality holds if and only if $\operatorname{Tr} A_{a}^{2}=\operatorname{Tr} A_{b}^{2}=\operatorname{Tr} A_{b}^{2}$ for $a, b=1, \cdots, p$ and either $A_{a}$ are all zero except possibly one of them or $A_{a}$ are all zero except two of them, say $A_{1}$ and $A_{2}$, and they can be transformed simultaneously by an orthogonal matrix into scalar multiple of the matrices of the form (3.15). Thus we obtain from (3.10) the inequality

$$
A(x) \geqq \sum_{a} \sum_{i<j}\left(\lambda_{i}^{(a)}-\lambda_{j}^{(a)}\right)^{2} K_{i j}^{(a)}-\frac{p-1}{p}\langle\beta, \beta\rangle^{2}(x) .
$$

Assume now that the scalar curvatures of $M$ are bounded below by a positive constant $d$. Then

$$
\sum_{a} \sum_{i<j}\left(\lambda_{i}^{(a)}-\lambda_{j}^{(a)}\right)^{2} K_{i j}^{(a)} \geqq d \sum_{a} \sum_{i<j}\left(\lambda_{i}^{(a)}-\lambda_{j}^{(a)}\right)^{2}
$$

and

$$
\begin{aligned}
& \sum_{i<j}\left(\lambda_{i}^{(a)}-\lambda_{j}^{(a)}\right)^{2}=(n-1) \operatorname{Tr} A_{a}^{2}-2 \sum_{i<j} \lambda_{i}^{(a)} \lambda_{j}^{(a)} \\
& -2 \sum_{i<j} \lambda_{i}^{(a)} \lambda_{j}^{(a)}=\operatorname{Tr} A_{a}^{2}-\left(\operatorname{Tr} A_{a}\right)^{2}
\end{aligned}
$$

and hence

$$
\sum_{a} \sum_{i<j}\left(\lambda_{i}^{(a)}-\lambda_{j}^{(a)}\right)^{2}=n\langle\beta, \beta\rangle(x)-n^{2}\langle\eta, \eta\rangle(x),
$$

where $\eta$ denotes the mean curvature normal of $M$. Thus we get the following inequality

$$
A \geqq\left(d n-\frac{p-1}{p}\langle\beta, \beta\rangle\right)\langle\beta, \beta\rangle-d n^{2}\langle\eta, \eta\rangle
$$

at each point of $M$.

We obtain from Corollaries 1 and 2 of Theorem 1 and Theorem 2 the following

Theorem 3. Let $M$ be an n-dimensional, Riemannian manifold with sectional curvatures bounded below by a positive constant $d$. Assume that $M$ is immersed in a Riemannian manifold $M^{\prime}$ of constant sectional curvature of dimension $n+p$ and that $M$ has a constant mean curvature. Then, if $M$ is compact and orientable or if the length of the second fundamental form $\beta$ of $M$ is constant, then we have 


$$
0 \geqq A \geqq\left\{d n-\frac{p-1}{p}\langle\beta, \beta\rangle\right\}\langle\beta, \beta\rangle-d n^{2}\langle\eta, \eta\rangle
$$

at each point of $M$, where $\eta$ denotes the mean curvature normal of $M$ which is parallel and $\langle\eta, \eta\rangle$ is a constant.

Now assume $M$ is compact and oriented and let $k=\langle\eta, \eta\rangle$. Then integrating both sides of the inequality (3.18) we obtain

$$
d n^{2} k \int_{M} * 1 \geqq \int_{M}\left\{d n-\frac{p-1}{p}\langle\beta, \beta\rangle\right\}\langle\beta, \beta\rangle * 1
$$

and we have the equality here if and only if

$$
d n^{2} k=\left\{d n-\frac{p-1}{p}\langle\beta, \beta\rangle\right\}\langle\beta, \beta\rangle
$$

and this implies also that $A=0$ and that $\beta$ is parallel by Theorem 1 . Then $\langle\beta, \beta\rangle$ must satisfy the quadratic equation $(p-1) x^{2}-p d n x+p n^{2} k=0$ and since the discriminant of this equation should be positive we should have the inequality

$$
d \geqq \frac{4 k(p-1)}{p} .
$$

\section{University of Notre Dame}

\section{Bibliography}

[1] S. S. Chern, M. DoCarmo and S. Kobayashi: Minimal submanifolds of a sphere with second fundamental form of constant length, to appear.

[2] J. A. Erbacher: Isomertric immersions of Riemannian manifold into space forms, Thesis, Brown University, 1970.

[3] S. Kobayashi and K. Nomizu: Foundations of Differential Geometry, Vol. I and II, J. Wiley, 1963 and 1969.

[4] K. Nomizu and B. Smyth: A formula of Simon's type and hypersurfaces of constant mean curvature. J. Differential Geometry 3 (1969), 367-377.

[5] J. Simons: Minimal varieties in Riemannian manifolds, Ann. of Math. 88 (1968), 62-105. 
